\title{
Analytical Algorithms to Quantify the Uncertainty in Remaining Useful Life Prediction
}

\author{
Shankar Sankararaman \\ SGT Inc., NASA Ames Research Center \\ Moffett Field, CA 94035, USA. \\ Ph: 650-604-0552 \\ shankar.sankararaman@nasa.gov \\ Abhinav Saxena \\ SGT Inc., NASA Ames Research Center \\ Moffett Field, CA 94035, USA. \\ Ph: 650-604-3208 \\ abhinav.saxena@nasa.gov
}

\author{
Matthew Daigle \\ NASA Ames Research Center \\ Moffett Field, CA 94035, USA. \\ Ph: 650-604-4583 \\ matthew.j.daigle@nasa.gov \\ Kai Goebel \\ NASA Ames Research Center \\ Moffett Field, CA 94035, USA. \\ Ph: 650-604-4204 \\ kai.goebel@nasa.gov
}

\begin{abstract}
This paper investigates the use of analytical algorithms to quantify the uncertainty in the remaining useful life (RUL) estimate of components used in aerospace applications. The prediction of RUL is affected by several sources of uncertainty and it is important to systematically quantify their combined effect by computing the uncertainty in the RUL prediction in order to aid risk assessment, risk mitigation, and decision-making. While sampling-based algorithms have been conventionally used for quantifying the uncertainty in RUL, analytical algorithms are computationally cheaper and sometimes, are better suited for online decision-making. While exact analytical algorithms are available only for certain special cases (for e.g., linear models with Gaussian variables), effective approximations can be made using the first-order second moment method (FOSM), the first-order reliability method (FORM), and the inverse first-order reliability method (Inverse FORM). These methods can be used not only to calculate the entire probability distribution of RUL but also to obtain probability bounds on RUL. This paper explains these three methods in detail and illustrates them using the state-space model of a lithium-ion battery.
\end{abstract}

\section{Table of Contents}

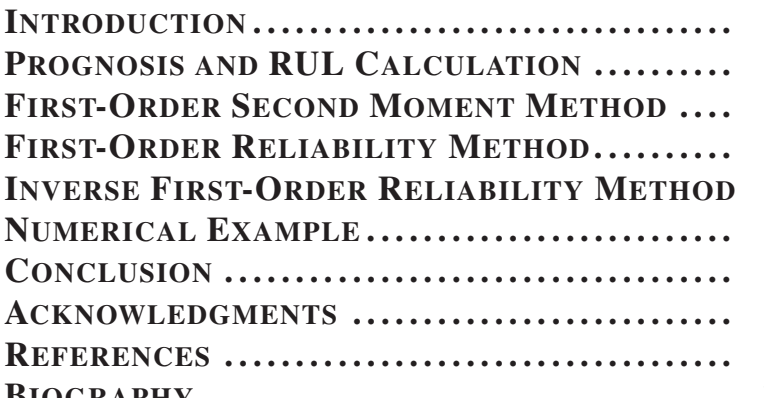

\section{INTRODUCTION}

The need for an accurate and efficient health management system has become exceedingly important in safety-critical and mission-critical aerospace systems. The most important goal of health management is to constantly monitor the performance of these aerospace systems, identify faults (diagnosis), predict possible failures in the near future, and quantify the remaining useful life (prognosis) in order to aid

978-1-4673-1813-6/13/\$31.00 (c) 2013 IEEE.

1 IEEEAC Paper \#2336, Version 1, Updated 01/3/2013. online decision-making. Sometimes, it may be challenging to perform health monitoring on the whole system due to its sheer complexity, and therefore, diagnosis and prognosis need to be performed on individual components that constitute the overall system. In this approach, mathematical models are developed for individual components, and then the component models are integrated to form the overall system. These component-level mathematical models can be constructed either using laws of physics (physics-based models [1]) or using data collected through component-level testing (data driven models [2]), and are used for both systemlevel diagnostics [3] and prognostics [4].

Uncertainty management is an important aspect of health monitoring, due to the presence of several unknown factors that affect the operations of the system of interest. Therefore, it is not only important to develop robust algorithms for diagnosis and prognosis, i.e., accurately perform diagnosis and prognosis in the presence of uncertainty, but also important to quantify the amount of confidence in the results of diagnosis and prognosis. This can be accomplished by quantifying the uncertainty in fault diagnosis and prognosis (future performance prediction and remaining useful life). It is important to perform such uncertainty quantification (UQ) online so as to enable in-flight decision-making capabilities. Sankararaman and Mahadevan [5,6] developed statistical (both frequentist and Bayesian) approaches to quantify the uncertainty in the three steps of diagnosis (detection, isolation, estimation) in an online health monitoring framework. There have also been a few papers [7-10] which discuss uncertainty propagation in prognosis; however, many of these papers are either suitable only for offline prognosis or they do not provide a comprehensive treatment of uncertainty. For example, the "Damage Prognosis Project" at Los Alamos National Laboratory [9] dealt with prognosis mainly in the context of offline testing and decision-making. Guan et al. [11] investigated Bayesian and maximum relative entropy methods for continuously updating the uncertainty in damage assessment, in the context of offline prognosis. Often, all the different sources of uncertainty - physical variability, data uncertainty, and model uncertainty - are not rigorously accounted for during prognosis; while most studies focus only on parameter variability and loading variability, the other sources of uncertainty are ignored. Sankararaman et al. [7] explained and addressed this issue in detail by identifying and accounting for the different types of uncertainty, the methodology was still developed in the context of offline testing. Therefore, there are several challenges relating to the topic of prognostics uncertainty quantification, and it is clear 
that further research is necessary in this regard. The rest of the paper focuses on this aspect, i.e., quantifying the uncertainty in the reamining useful life (RUL) prediction.

This paper pursues a model-based framework for prognosis and RUL calculation. Since state-space models are suitable for representing time-dependent behavior, the governing differential equations of the component of interest are transformed into equivalent state space representations. There are three major challenges before prognosis can be performed and the RUL can be calculated.

1. State Uncertainty: The first challenge is to estimate the current state of the system and the associated uncertainty. Typically, this problem is formulated as an estimation problem or a state identification problem, and filtering techniques such as particle filtering [12] are used. Though the true state at any time instant is a deterministic quantity, i.e., it is not precisely known and in most cases, impossible to estimate with certainty due to (i) the presence of measurement errors and sensor noise (data uncertainty) in the data collected for estimation; (ii) uncertainty in the initial state; (iii) the noise in the state space model used for estimation; and (iv) model uncertainty. Filtering techniques such as particle filtering are based on the Bayesian philosophy of uncertainty representation, according to which randomness can be perceived as lack of information/precision [13], and can be used to represent even an inherently deterministic quantity (which does not exhibit natural variabililty but is not known precisely) using a probability distribution.

2. Future Loading Uncertainty: In practical engineering systems, it is almost impossible to accurately predict the future loading and environmental conditions. Following the Bayesian approach, future loading needs to be represented as an uncertain quantity. An important challenge is to quantify the amount of uncertainty in future loading. Though a few publications have addressed this issue [14, 15], further research is needed in this direction. However, the focus of the present paper is not on uncertainty characterization, and therefore it is assumed that future loading variability has been characterized (e.g., based on already existing loading profiles and data sets). After this uncertainty is characterized and quantified, it needs to be included in prognosis to quantify its effect on the uncertainty in RUL.

3. Process Noise: Process noise is an important component of model uncertainty, and commonly represented as a random variable which needs to be included while future predictions are made and the RUL is estimated. Though the process noise term can be estimated using the data collected during the estimation stage, the future process noise may be significantly different from the estimated noise, and cannot be known in advance. However, it is still necessary to assume statistical distributions for the process noise in the different states, and include these distributions in the calculation of RUL uncertainty.

The task of quantifying the uncertainty in the RUL prediction is primarily an uncertainty propagation problem [16], i.e., the above discussed different types of uncertainty need to be propagated through the state space model until the End of Life (EOL). Sampling-based methods (adaptive sampling, Latin hypercube sampling, Markov Chain Monte Carlo sampling, etc.) can be easily used to quantify the uncertainty in RUL, but they have two major disadvantages:
1. Several thousands of samples and hence, several thousands of prognostic evaluations may be necessary to accurately quantify the entire probability distribution of RUL, which may not be computationally feasible for online health monitoring. Theoretically, an infinite number of samples is necessary to accurately calculate the probability distribution of RUL. An increasingly smaller set of samples result in increasingly worse representation of the distribution.

2. The method of drawing random samples may not be preferred if repeatability is a desired criterion (for example, for online decision-making or also for certification of algorithms). In other words, the PDF of RUL depends on the exact set of considered samples; if the calculation is repeated with a different set of samples (which is likely the case if purely random samples are drawn), then a different (albeit only slightly, if the number of samples is large) probability distribution may be obtained. This difficulty can be overcome by predetermining the percentile values which can in turn be used to draw samples. In this case, it may be possible to preserve a particular statistic of the probability distribution, but it may be difficult to accurately obtain the entire probability distribution. For example, using the unscented transform sampling approach proposed by Daigle et al. [17], the mean and the variance of RUL can be efficiently calculated using a few samples. While it is important to calculate the first two moments of RUL, it is equally important to estimate the tail probabilities of the distribution of RUL, because failure is often caused due to events related to the tails of probability distributions. In other words, in a well-designed system, the probability of failure is very small (usually of the order of $10^{-3}$ or less), and in order to compute the value of RUL which corresponds to such low probabilities, it is important to accurately estimate the tail of the probability distribution of RUL.

This paper investigates the use of analytical algorithms for calculating the uncertainty in RUL, as an alternative to sampling-based methods. Several analytical algorithms such as the first-order second moment method (FOSM), first-order reliability method (FORM), second-order reliability method (SORM), etc. have been used to calculate the reliability of structural systems, and they can also be used for uncertainty propagation. This paper investigates the use of the FOSM and FORM methods using state space models, and extends them to calculate the uncertainty in RUL prediction. These methods not only require very few prognostic evaluations in comparison with the sampling-based approaches but can also produce repeatable calculations, i.e., the exact same PDF on every repetition of the algorithm. While the former directly aids in online prognosis since fewer evaluations would lead to quicker calculations, it is worth noting that the latter feature is an important criterion for existing verification, validation, and certification protocols in the aerospace domain. Therefore, investigating such analytical algorithms allows us to move a step closer towards adopting prediction algorithms (which are inherently stochastic), by meeting the needs of the current certification process.

The rest of the paper is organized as follows. Section 2 explains the state space model formulation and describes how the estimation of uncertainty in RUL can be viewed as an uncertainty propagation problem. Sections 35 present the different analytical methods for quantifying the uncertainty in the RUL prediction. Finally, the methods are illustrated using the state space model of a lithium-ion battery model in Section 6, and Section 7 concludes the paper. 


\section{Prognosis and RUL Calculation}

The section formulates the prognosis problem, and explains the calculation of Remaining Useful Life [4]. The various sources of uncertainty are explained, and finally, it is illustrated how the calculation of uncertainty in RUL can be treated as an uncertainty propagation problem.

To begin with, consider the state space model which is used to continuously predict the state of the system, and therefore, aid in prognosis. Consider a generalized state space model as:

$$
\dot{\mathbf{x}}(t)=\mathbf{f}(t, \mathbf{x}(t), \boldsymbol{\theta}(t), \mathbf{u}(t), \mathbf{v}(t))
$$

where $\mathbf{x}(t) \in \mathbb{R}^{n_{x}}$ is the state vector, $\boldsymbol{\theta}(t) \in \mathbb{R}^{n_{\theta}}$ is the parameter vector, $\mathbf{u}(t) \in \mathbb{R}^{n_{u}}$ is the input vector, $\mathbf{v}(t) \in \mathbb{R}^{n_{v}}$ is the process noise vector, $\mathbf{f}$ is the state equation. Typically, all of these quantities are uncertain in reality.

The state vector at time $t$, i.e., $\mathbf{x}(\mathbf{t})$ (and the parameters $\boldsymbol{\theta}(t)$, if they are unknown) is (are) typically estimated using filtering approaches until the time $t$ when data is available. Let $\mathbf{y}(t) \in \mathbb{R}^{n_{y}}, \mathbf{n}(t) \in \mathbb{R}^{n_{n}}$, and $\mathbf{h}$ denote the output vector, measurement noise vector, and output equation respectively. Then,

$$
\mathbf{y}(t)=\mathbf{h}(t, \mathbf{x}(t), \boldsymbol{\theta}(t), \mathbf{u}(t), \mathbf{n}(t))
$$

Note that the output equation or data is not used in the prognosis stage, since the focus is on predicting the future and the associated uncertainty.

Prognostics and RUL prediction are concerned with the performance of the component that lies outside a given region of acceptable behavior. The desired performance is expressed through a set of $n_{c}$ constraints, $C_{E O L}=\left\{c_{i}\right\}_{i=1}^{n_{c}}$, where $c_{i}: \mathbb{R}^{n_{x}} \times \mathbb{R}^{n_{\theta}} \times \mathbb{R}^{n_{u}} \rightarrow \mathbb{B}$ maps a given point in the joint state-parameter space given the current inputs, $(\mathbf{x}(t), \boldsymbol{\theta}(t), \mathbf{u}(t))$, to the Boolean domain $\mathbb{B} \triangleq[0,1]$, where $c_{i}(\mathbf{x}(t), \boldsymbol{\theta}(t), \mathbf{u}(t))=1$ if the constraint is satisfied, and 0 otherwise.

These individual constraints may be combined into a single threshold function $T_{E O L}: \mathbb{R}^{n_{x}} \times \mathbb{R}^{n_{\theta}} \times \mathbb{R}^{n_{u}} \rightarrow \mathbb{B}$, defined as:

$$
T_{E O L}(\mathbf{x}(t), \boldsymbol{\theta}(t), \mathbf{u}(t))= \begin{cases}1, & 0 \in\left\{c_{i}(\mathbf{x}(t), \boldsymbol{\theta}(t), \mathbf{u}(t))\right\}_{i=1}^{n_{c}} \\ 0, & \text { otherwise }\end{cases}
$$

$T_{E O L}$ is equal to 1 when any of the constraints are violated. Then, the End of Life (EOL, denoted by $E$ ) at any time instant $t_{P}$ is then defined as the earliest time point at which this occurs:

$$
E\left(t_{P}\right) \triangleq \inf \left\{t \in \mathbb{R}: t \geq t_{P} \wedge T_{E O L}(\mathbf{x}(t), \boldsymbol{\theta}(t), \mathbf{u}(t))=1\right\}
$$

The Remaining Useful Life (RUL, denoted by $R$ ) at time instant $t_{P}$ is expressed as:

$$
R\left(t_{P}\right) \triangleq E\left(t_{P}\right)-t_{P}
$$

Thus, it is clear that RUL predicted at time $t_{P}$, i.e., $R\left(t_{P}\right)$ depends on

1. Present time $\left(t_{P}\right)$

2. Present state estimate $\left(\mathbf{x}\left(t_{P}\right)\right)$; using the present state estimate and the state space equations in Eq. 1, the future states $\left(\mathbf{x}\left(t_{P}\right), \mathbf{x}\left(t_{P}+1\right), \mathbf{x}\left(t_{P}+2\right), \ldots, \mathbf{x}\left(t_{P}+R\left(t_{P}\right)\right)\right)$ can be calculated.
3. Future loading $\left(\mathbf{u}\left(t_{P}\right), \mathbf{u}\left(t_{P}+1\right), \mathbf{u}\left(t_{P}+2\right), \ldots, \mathbf{u}\left(t_{P}+\right.\right.$ $\left.R\left(t_{P}\right)\right)$ ); these values are needed to calculate the future state values using the state space equations. In this paper, constant amplitude loading is considered, and the amplitude is assumed to follow a particular probability distribution. Future work will consider variable amplitude loading.

4. Parameters $\left(\boldsymbol{\theta}\left(t_{P}: t_{P}+R\left(t_{P}\right)\right)\right)$; the values of parameters are also needed to calculate the future states. If measurement data are available, then the parameters can be estimated along with the states using filtering techniques. However, this paper addresses only the prognosis problem (future prediction), and it is assumed that measurement data is not available after time $t_{P}$. Therefore, the parameters $\boldsymbol{\theta}\left(t_{P}: t_{P}+R\left(t_{P}\right)\right)$ need to be assumed in order to calculate future states and estimate the RUL prediction. In this paper, for the sake of illustration, the parameters are assumed to be constants (over time) and precisely known.

5. Process noise $\left(\mathbf{v}\left(t_{P}\right), \mathbf{v}\left(t_{P}+1\right), \mathbf{v}\left(t_{P}+2\right), \ldots, \mathbf{v}\left(t_{P}+\right.\right.$ $\left.R\left(t_{P}\right)\right)$ ).

The focus of this paper is to quantify the uncertainty in RUL as a result of the uncertainties in these quantities. Note that the dependence of $R\left(t_{P}\right)$ on other uncertain quantities is implicit, i.e., the RUL depends on the process noise and loading, and in turn, how long the process noise and the future loading need to be considered during prognosis directly depends on the RUL itself. Therefore, it is challenging to employ the analytical methods for uncertainty propagation, since an explicit function, which establishes a one-to-one relationship from the various sources of uncertainty to the RUL, is necessary. The constant value of the input loading amplitude is denoted by $u_{E}$, and can be easily included as an input to this explicit function. However, the inclusion of process noise is not straightforward because it is necessary to include the process noise at all time instants as inputs; this increases the dimensionality of the problem several fold. Further, in some cases where the sensitivity of the uncertainty in RUL to the uncertainty in the process noise is negligible, it is meaningful to ignore the contribution of process noise uncertainty. Therefore, process noise is not considered in the present paper and the one-to-one function can be written as:

$$
R\left(t_{P}\right)=G\left(\mathbf{x}\left(t_{P}\right), \mathbf{u}^{E}\right)
$$

Given a realization of $\mathbf{x}\left(t_{P}\right)$ and $\mathbf{u}^{E}$, the above equation is continuously evaluated using the state space model in Eq. 1, and $R\left(t_{P}\right)$ is computed when end of life is reached. In a sampling-based procedure, this is repeated for several thousands of samples of $\mathbf{x}\left(t_{P}\right)$ and $\mathbf{u}^{E}$, the corresponding realization of $R\left(t_{P}\right)$ are calculated to obtain a histogram, which can be used to construct the probability density function (PDF) of $R\left(t_{P}\right)$. As stated earlier, such sampling-based approaches may be computationally expensive, and this paper investigates computationally efficient, analytical alternatives to construct the PDF. In general, there may be other sources of uncertainty (e.g., parameter uncertainty), and hence, the above function $G$ may have several inputs. The following sections discuss the analytical methods for a generic number of inputs, as:

$$
R=G(\boldsymbol{X})
$$

where $\boldsymbol{X}$ is a vector $\boldsymbol{X}=\left\{X_{1}, X_{2}, \ldots X_{i}, \ldots X_{n}\right\}$, where $n$ is the length of the vector $\boldsymbol{X}$, and therefore the number of uncertain "inputs" to $G$. For example, if there are two states (their values at time $t_{P}$ are uncertain) and one loading amplitude (that is uncertain), then $n=3$. Each $X_{i}$ has its own probability distribution and it is desired to calculate the probability distribution of $R$. If these probability distributions 
are Gaussian and if $G$ is a linear function, then uncertainty propagation through $G$ is simple and closed-form expressions are available to estimate the statistics of $R$. In general, the state-space equation, i.e., Eq. 1, and therefore, damage progression equations are non-linear; further, the threshold function in Eq. 3 may also be non-linear. Therefore, $G$ is non-linear in several practical cases, and thus the uncertainty quantification methodology must be applicable to the general case, i.e, non-linearity in $G$ and non-normality in $\boldsymbol{X}$.

\section{First-Order Second Moment Method}

The first-order second moment approach, as the name suggests, is a simple approximation of $R$ using First-order Taylor series expansion. The first two moments, i.e. mean $\left(\mu_{\boldsymbol{X}}\right)$ and variance $\left(\sigma_{\boldsymbol{X}}\right)$ of $\boldsymbol{X}$ are used to approximate the first two moments of $R$. Consider the first-order Taylor series expansion of $Y=G(\boldsymbol{X})$ around $\mu_{\boldsymbol{X}}$, as:

$$
R=G\left(\mu_{\boldsymbol{X}}\right)+\sum_{i=1}^{i=n}\left(X_{i}-\mu_{X_{i}}\right)\left(\frac{\partial R}{\partial X_{i}}\right)_{\mu_{\boldsymbol{X}}}
$$

Note that $R$ is a linear function of $\boldsymbol{X}$ with the partial derivatives as coefficients, and therefore, it is straightforward to approximate the mean and variance of $R$, as:

$$
\begin{gathered}
\mu_{R}=G\left(\mu_{\boldsymbol{X}}\right) \\
\sigma_{R}^{2}=\sum_{i=1}^{i=n} \sum_{j=1}^{j=n}\left(\frac{\partial R}{\partial X_{i}}\right)_{\mu_{\boldsymbol{X}}}\left(\frac{\partial R}{\partial X_{j}}\right)_{\mu_{\boldsymbol{X}}} \operatorname{Cov}\left(X_{i}, X_{j}\right)
\end{gathered}
$$

When the inputs to $G$ are uncorrelated, then the expression for variance in Eq. 10 simplifies to:

$$
\sigma_{R}^{2}=\sum_{i=1}^{i=n}\left(\frac{\partial R}{\partial X_{i}}\right)_{\mu_{X}}^{2} \sigma_{X_{i}}^{2}
$$

Note that the first-order second moment approach can only compute the mean and variance of the remaining useful life $(R)$ prediction. It is not directly useful in estimating the type of probability distribution of $R$, and therefore cannot be used to calculate tail probabilities. However, if each of the inputs $X_{i}$ were to be normally distributed (and independent of each other), then it can be easily proved that their linear combination is also a normally distributed variable [18]. This observation is used to develop a general methodology to calculate the entire CDF of the Remaining Useful Life $(R)$ prediction in the following stages:

1. FORM for Normal Variables: First, consider the case when the variables are normal. Even in this case, the above method is suitable only when $G$ is linear. When $G$ is nonlinear, it is not appropriate to always calculate the gradient at the mean $\mu_{\boldsymbol{X}}$. This leads to obvious question - "where to linearize"? The "location of linearization" is chosen analytically, and then used to calculate the CDF of RUL at a particular $R=r$, i.e., $F_{R}(R=r)=P(R \leq r)$. Note that an upper case letter refers to the name of a random variable whereas the corresponding lower case letter refers to its realization. It will be illustrated that the location (point) of linearization varies with the choice of $r$. By repeating the entire process for different choices of $r$, the entire CDF can be calculated. This method, known as the first-order reliability method (FORM), is discussed in the first part of Section 4.
2. FORM for Non-Normal Variables: The above FORM method for normal variables is extended to non-normal variables in the latter part of Section 4.

3. Inverse FORM: While Section 4 deals with calculating the CDF value for a given realization of $R$, Section 5 considers the inverse problem, i.e. calculating the realization of $R$ that corresponds to a given CDF value. This method, popularly known as the Inverse CDF approach, can be used to calculate probability bounds on the remaining useful life prediction, which is useful for decision-making.

\section{First-Order REliability Method}

The first-order reliability method (FORM) was originally developed by structural engineers in order to estimate the reliability of structural systems. The contribution of this paper is to extend this method to state space models and health monitoring with the goal of computing the probability distribution of Remaining Useful Life. Though the estimation of reliability and the calculation of $\mathrm{CDF}$ are statistically equivalent, the FORM method is described from a purely uncertainty propagation perspective, without reference to reliability calculation.

Consider Eq. 7, which expresses the RUL as a function of the various sources of uncertainty. The first subsection explains the FORM method when $\boldsymbol{X}$ consists of normally distributed variables and the next subsection extends the method to the non-normal case.

\section{Normal Variables}

The goal of the FORM approach is to calculate the CDF value, i.e. $F_{R}(r)=P(R \leq r)$, given $r$ which is a realization of the random variable $R$. In this subsection, assume that the $i^{\text {th }}$ input $X_{i}$ is normally distributed as $\mathrm{N}\left(\mu_{X_{i}}, \sigma_{X_{i}}\right)$.

FORM achieves the aforementioned goal by approximating the non-linear equation $R=G(\boldsymbol{X})$ using a linear equation in order to easily compute the CDF of $R$. The linear equation is constructed using a Taylor series approximation around the so-called "point of linearization". The difference in FORM (with respect to FOSM) is that the point of linearization varies from one choice of $r$ to another. The identification of the point of linearization is the most important component of the FORM algorithm.

In order to calculate $F_{R}(r)=P(R \leq r)$, consider the contours of the function $R-r=G(\boldsymbol{X})-r$; in particular consider the curve described by $G(\boldsymbol{x})-r=0$, where $\boldsymbol{x}$ is a realization of $\boldsymbol{X}$. This curve differentiates the multidimensional space into two regions: one region where $R<r$, and another region where $R>r$, as shown in Fig. 1. (This curve of demarcation is popularly called as the limit state in reliability analysis. The equation corresponding to the limit state divides the space into two zones - zone of failure and zone of safety, respectively. This terminology is not used in this paper because FORM is now used for uncertainty propagation rather than reliability calculation.)

Any point lying on the curve of demarcation would satisfy the equation $R=G(\boldsymbol{X})$. Since (1) this curve serves as the demarcation between the two zones given by $R>r$ and $R<r$; and (2) it is of interest to calculate the probability $P(R \leq r)$, it is intuitive that it is important to identify a linear function which closely resembles the contour $G(\boldsymbol{x})-r=0$. Hence, the point of linearization must lie on this curve of demarcation; in other words, the point of linearization must 


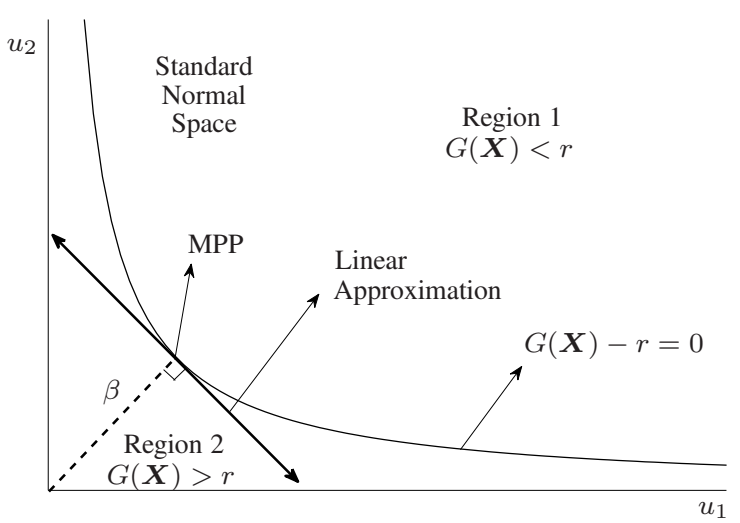

Figure 1: Estimating MPP in FORM

satisfy the equation $G(\boldsymbol{x})-r=0$. This is clearly different from the FOSM approach, where the mean $\mu_{\boldsymbol{X}}$ was chosen as the point of linearization; for an arbitrary choice of $r$, it is obvious that the mean $\mu_{\boldsymbol{X}}$ will not satisfy this equation.

Therefore, the point of linearization should be located on the curve of demarcation. However, there are infinite points that satisfy this criterion, and it is important to select the appropriate one. Each of these infinite points has a likelihood of occurrence, and intuitively, the point of maximum likelihood is chosen as the point of linearization. This likelihood can be calculated using the probability density function of the underlying random variables. For a single normal random variable $X$ with mean $\mu$ and standard deviation $\sigma$, the PDF is given by:

$$
f_{X}(x \mid \mu, \sigma)=\frac{1}{\sigma \sqrt{2 \pi}} \exp \left[-\frac{(x-\mu)^{2}}{2 \sigma^{2}}\right] .
$$

For example, when $\mu=10$ and $\sigma=1, x=10$ is 1.65 times more likely to occur than $x=9$. The maximum value of the likelihood function occurs at $x=\mu$; therefore, the farther $x$ is away from the mean $\mu$, the lower the likelihood of occurrence of $x$. As explained earlier, the mean cannot be chosen as the point of linearization since $G(\mu)-r \neq 0$. Therefore, if there is a single input variable, the point of linearization is chosen in such a way that it satisfies the equation $G(x)-r=0$ and the value of $\|x-\mu\|$ is minimum.

However, in a general uncertainty propagation problem, the input to $G$ is a vector, i.e., $\boldsymbol{X}=\left\{X_{1}, X_{2}, \ldots X_{i}, \ldots X_{n}\right\}$, and each $X_{i}$ has its own mean $\mu_{X_{i}}$ and standard deviation $\sigma_{X_{i}}$. The objective is to identify the point of maximum likelihood, which can be calculated by maximizing the joint probability density function of all the input random variables. If the variables are independent, then the joint density function of $\boldsymbol{X}$ is expressed as:

$$
f_{\boldsymbol{X}}(\boldsymbol{x})=\prod_{i=1}^{i=n} \frac{1}{\sigma_{i} \sqrt{2 \pi}} \exp \left[-\frac{\left(x_{i}-\mu_{i}\right)^{2}}{2 \sigma_{i}^{2}}\right] .
$$

It can be verified (by taking the logarithm) that the maximizer of the above function simultaneously minimizes

$$
\beta=\sum_{i=1}^{i=n}\left(\frac{x_{i}-\mu_{i}}{\sigma_{i}}\right)^{2} .
$$

Eq. 14 can rewritten as:

$$
\beta=\sum_{i=1}^{i=n} u_{i}^{2}
$$

where

$$
u_{i}=\frac{x_{i}-\mu_{i}}{\sigma_{i}} .
$$

If the above computation were performed for every realization $x_{i}$ of the random variable $X_{i}$, then the corresponding $u_{i}$ s would be realizations of the standard normal variable $U_{i}$, i.e., $U_{i} \sim N(0,1)$. Therefore, Eq. 16 is referred to as the standard normal transformation. In the space of standard normal variables, maximizing the likelihood of occurrence is equivalent to minimizing Eq. 15, which implies that the point of linearization is that point on the curve of demarcation, whose distance (measured in the standard normal space) from the origin is minimum. Since the point of linearization has the maximum likelihood of occurrence, it is popularly known as the Maximum Probable Point (MPP), as indicated in Fig. 1. The Maximum Probable Point, therefore, represents the minimum distance (measured from the origin, in the standard normal space), and this minimum distance is of significance, because it can be proved that:

$$
P(R \leq r)=\Phi(-\beta)
$$

where $\Phi($.$) represents the standard normal cumulative dis-$ tribution function. In order to prove Eq. 17, consider the first order Taylor's series expansion of $Z=G(\boldsymbol{X})-r$ by linearizing around the MPP. Then, $Z$ is approximated to be a normal random variable; its CDF value measured at $Z=0$ is exactly equal to $P(R \leq r)$, and is calculated as:

$$
P(Z \leq 0)=P(R \leq r)=\Phi\left(-\frac{\mu_{Z}}{\sigma_{Z}}\right)
$$

Using the Taylor series expansion in Eq. 8, it can be proved that [18]:

$$
\beta=\sum_{i=1}^{i=n} u_{i}^{2}=\frac{\mu_{Z}}{\sigma_{Z}}
$$

Therefore, the problem of calculating the CDF reduces to identifying the MPP on the curve of demarcation. This can be posed as a constrained optimization problem, as follows:

$$
\begin{gathered}
\text { Minimize } \boldsymbol{u}^{T} \boldsymbol{u} \\
\text { s.t. } \stackrel{G}{G}(\boldsymbol{x})=r \\
\boldsymbol{u}=\left\{u_{1}, u_{2} \ldots u_{i}, \ldots u_{n}\right\} \\
u_{i}=\left(x_{i}-\mu_{i}\right) / \sigma_{i} \\
(i=1 \text { to } n)
\end{gathered}
$$

The above optimization problem can be solved using the Rackwitz-Fiessler [19] algorithm, an iterative procedure, as follows:

1. Initialize counter $j=0$ and start with an initial guess for the Most probable point (MPP), i.e., $\boldsymbol{x}^{j}=\left\{x_{1}^{j}, x_{2}^{j}, \ldots x_{i}^{j}, \ldots x_{n}^{j}\right\}$, a column vector.

2. Transform into standard normal space and calculate $\boldsymbol{u}^{j}=\left\{u_{1}^{j}, u_{2}^{j}, \ldots u_{i}^{j}, \ldots u_{n}^{j}\right\}$ using Eq. 16 , a column vector.

3 . Compute the gradient vector in the standard normal space, i.e., $\boldsymbol{\alpha}=\left\{\alpha_{1}, \alpha_{2}, \ldots \alpha_{n}\right\}$, another column vector where

$$
\alpha_{i}=\frac{\partial g}{\partial u_{i}}=\frac{\partial g}{\partial x_{i}} \times \frac{\partial x_{i}}{\partial u_{i}}=\frac{\partial g}{\partial x_{i}} \times \sigma_{i}
$$


4. In the iterative procedure, the next point $u^{j+1}$ is calculated using a Newton-Raphson type equation, as:

$$
\boldsymbol{u}^{j+1}=\frac{1}{\|\boldsymbol{\alpha}\|}\left[\boldsymbol{\alpha}^{T} \boldsymbol{u}^{j}-G\left(\boldsymbol{x}^{j}\right)\right] \frac{\boldsymbol{\alpha}}{\|\boldsymbol{\alpha}\|}
$$

5. Transform back into original space, i.e., compute $x^{j+1}$, and continue starting from Step 3 until the iterative procedure converges. Using tolerance limits $\delta_{1}$ and $\delta_{2}$, convergence can be checked verified if the following two criteria are satisfied: (i) the point lies on the curve of demarcation, i.e., $\left|G\left(\boldsymbol{x}^{j}\right)-r\right| \leq \delta_{1}$; and (2) the solution does not change between two iterations, i.e., $\left|\boldsymbol{x}^{j+1}-\boldsymbol{x}^{j}\right| \leq \delta_{2}$.

The above described iterative procedure usually converges within 4 or 5 iterations. In each iteration, the transformation to the standard normal space is straightforward only when the variables are originally normal. Therefore, the method needs to be modified to include non-normal variables, as explained in the next subsection.

\section{Extension to Non-normal Variables}

Now consider the case where the inputs $X_{i}(i=1$ to $n)$ have arbitrary probability distributions given by their CDFs as $F_{X_{i}}\left(x_{i}\right)(i=1$ to $n)$. Now that $X_{i}$ is not normally distributed, Eq. 16 cannot be used for standard normal transformation. Therefore, it is necessary to calculate $u_{i}$ from a given $x_{i}$ meaningfully, so that $u_{i}$ represents a realization of the standard normal variable. One simple transformation is based on probability integral transform concept, as:

$$
u_{i}=\Phi^{-1}\left(F_{X_{i}}\left(X_{i}=x_{i}\right)\right)
$$

where $\Phi^{-1}($.$) refers to the inverse of the standard normal$ distribution function [18].

In addition to the above procedure, there are also other transformation techniques. For example, a two-parameter transformation procedure estimates the mean $\mu_{i}$ and standard deviation $\sigma_{i}$ of the normal distribution by equating the CDF and PDF values of the distribution of $X$ and the normal distribution. Then, Eq. 16 can be used to calculate $u_{i}$ from $x_{i}$. Note that the mean $\mu_{i}$ and standard deviation $\sigma_{i}$ are dependent on the value of $x_{i}$. Similarly, Chen and Lind [20] proposed a three-parameter transformation procedure by introducing a third parameter, a scale factor which is estimated by matching the slope of the probability density function in addition to the PDF and CDF values. Further, when the inputs are correlated or statistically dependent, it is necessary to transform them to uncorrelated standard normal space. Haldar and Mahadevan [18] describe methods for such transformation. It must be noted that any transformation must be accompanied by suitably computing the derivatives in the standard normal space, and Eq. 21 must be appropriately replaced.

Note that above FORM procedure calculates the CDF value at a particular value of RUL. It answers the question: What is the probability that the RUL is smaller than a given number? This question is answered using a search procedure which computes the CDF value corresponding to a given RUL value, and this search is accomplished through an iterative procedure, like in an optimization algorithm. In order to obtain the entire CDF, the whole procedure is repeated with multiple values of RUL. Sometimes, it may not be possible to identify values of RUL in order to calculate the entire CDF because the spread of the distribution may not be known in advance. So, the next section discusses the Inverse FORM procedure, which answers the question: What is the value of RUL which corresponds to a given probability level? In other words, what is the $\alpha$-percentile (e.g., $5 \%, 95 \%$, etc.) value of RUL? By repeating this procedure for one lower percentile and one upper percentile value, the probability bounds on RUL can be calculated.

\section{INVERSE FIRST-ORDER RELIABILITY METHOD}

Given $\beta$ or $\lambda=\Phi(-\beta)$, the inverse FORM approach can be used to calculate $r$ such that $F_{R}(r)=P(R \leq r)=\lambda$. The theory behind inverse FORM is exactly the same as FORM, and the algorithm discussed in Section 4 is modified so that the CDF value can be specified and $r$ can be calculated. The various steps involved in the iterative procedure for Inverse FORM are outlined below:

1. Initialize counter $j=0$ and start with an initial guess for the Most probable point (MPP), i.e., $\boldsymbol{x}^{j}=\left\{x_{1}^{j}, x_{2}^{j}, \ldots x_{i}^{j}, \ldots x_{n}^{j}\right\}$.

2. Transform into standard normal space and calculate $\boldsymbol{u}^{j}=\left\{u_{1}^{j}, u_{2}^{j}, \ldots u_{i}^{j}, \ldots u_{n}^{j}\right\}$.

3. Compute the gradient vector in the standard normal space, i.e., $\boldsymbol{\alpha}=\left\{\alpha_{1}, \alpha_{2}, \ldots \alpha_{n}\right\}$, as explained in Section 4 .

4. In the iterative procedure, the next point $u^{j+1}$ is calculated as:

$$
\boldsymbol{u}^{j+1}=-\frac{\alpha}{|\alpha|} \beta
$$

5. Transform back into original space, i.e., compute $x^{j+1}$, and continue starting from Step 3 until the iterative procedure converges. Using tolerance limits $\delta_{1}$ and $\delta_{2}$, convergence can be checked verified if the following two criteria are satisfied: (i) the point lies on the curve of demarcation, i.e., $\left|G\left(\boldsymbol{x}^{j}\right)-r\right| \leq \delta_{1}$; and (2) the solution does not change between two iterations, i.e., $\left|\boldsymbol{x}^{j+1}-\boldsymbol{x}^{j}\right| \leq \delta_{2}$.

Similar to the Rackwitz-Fiessler algorithm, the above iterative procedure usually convergences within 4 or 5 iterations, and therefore is suitable for quick calculations.

As stated earlier, the Inverse FORM procedure is useful to calculate probability bounds. For example, by repeating the above algorithm for $\lambda=0.05$ and $\lambda=0.95$, it is possible to estimate the $90 \%$ probability bounds on the remaining useful life. In fact, the entire CDF can be constructed by repeating the analysis for several values of $\lambda$ $(0.05,0.1,0.2,0.3,0.4,0.5,0.6,0.7,0.8,0.9,0.95)$.

The advantage of Inverse FORM is that the number of prognostic evaluations is extremely small compared to samplingbased approaches. For the sake of illustration, consider that the above iterative algorithm converges in 5 iterations. Each iteration requires the computation of the gradient vector; if there are $k$ inputs, then $k+1$ computations are necessary for each evaluation - one for each derivative and one for the evaluation of $G$. Hence, the total number of prognostic evaluations is equal to $5(k+1)$. If the analysis is repeated for 2 values of $\lambda$ (in order to compute the probability bounds on RUL), then it takes $10(k+1)$ prognostic evaluations, which is more computationally efficient than sampling-based techniques, and, therefore suitable for online decision-making. 


\section{Numerical EXAMPLE}

The proposed methods are illustrated using a state space model of a lithium-ion battery, which is an important component in aerospace applications and which is also being studied at NASA Ames Research Center as part of the rover testbed [21]. Though the method is illustrated using a battery model, it is general and can be applicable to state space models in several engineering domains.

\section{Description of the Model}

This battery model, earlier used by Daigle et al. [17] for prognosis, is similar to the models presented by Chen and Rincon-Mora [22]. The model is based on an electrical circuit equivalent as shown in Fig. 2, where the large capacitance $C_{b}$ holds the charge $q_{b}$ of the battery. The $R_{C P^{-}}-C_{C P}$ pair captures the major nonlinear voltage drop due to concentration polarization, $R_{s}$ captures the so-called I-R drop, and $R_{p}$ models the parasitic resistance that accounts for selfdischarge. This relatively simple battery model is sufficient to capture the major dynamics of the battery, but ignores temperature effects and other minor battery processes.

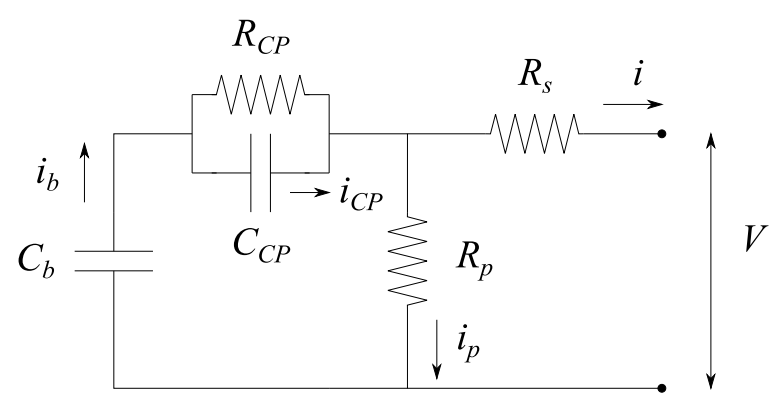

Figure 2: Battery equivalent circuit

The state-of-charge, $S O C$, is computed as

$$
S O C=1-\frac{q_{\max }-q_{b}}{C_{\max }}
$$

where $q_{b}$ is the current charge in the battery (related to $\left.C_{b}\right), q_{\max }$ is the maximum possible charge, and $C_{\max }$ is the maximum possible battery capacity (i.e., nominally, its rated capacity). The concentration polarization resistance is a nonlinear function of $S O C$ :

$$
R_{C P}=R_{C P 0}+R_{C P 1} \exp \left(R_{C P 2}(1-S O C)\right)
$$

where $R_{C P 0}, R_{C P 1}$, and $R_{C P 1}$ are empirical parameters. The resistance, and, hence, the voltage drop, increases exponentially as $S O C$ decreases [14].

Voltage drops across the individual circuit elements are given by

$$
\begin{aligned}
V_{b} & =q_{b} / C_{b} \\
V_{C P} & =q_{C P} / C_{C P} \\
V_{p} & =V_{b}-V_{C P}
\end{aligned}
$$

where $q_{C P}$ is the charge associated with the capacitance $C_{C P}$. The terminal voltage of the battery is

$$
V=V_{b}-V_{C P}-R_{s} i
$$

\begin{tabular}{cc}
\hline Parameter & Value \\
\hline$C_{b}$ & 9844 \\
$R_{s}$ & 0.143014 \\
$R_{p}$ & 500 \\
$C_{C P}$ & 70.3767 \\
$R_{C P 0}$ & 0.019829 \\
$R_{C P 1}$ & $3.68606 \times 10^{-14}$ \\
$R_{C P 2}$ & 31.9213 \\
$q_{\max }$ & 41400 \\
$C_{\max }$ & 6900 \\
\hline
\end{tabular}

Table 1: Battery Model Parameters

where $i$ is the battery current at the terminals. Currents associated with the individual circuit elements are given by

$$
\begin{aligned}
i_{p} & =V_{p} / R_{p} \\
i_{b} & =i_{p}+i \\
i_{C P} & =i_{b}-V_{C P} / R_{C P}
\end{aligned}
$$

The charges are then governed by

$$
\begin{aligned}
\dot{q}_{b} & =-i_{b} \\
\dot{q}_{C P} & =i_{C P}
\end{aligned}
$$

It is of interest to predict the end-of-discharge as defined by a voltage threshold $V_{E O D}$. So, $C_{E O L}$ consists of only one constraint:

$$
c_{1}: V>V_{E O D}
$$

The parameters of the battery model are assumed to be deterministic and are shown in Table 1. All voltages are measured in Volts, resistances are measured in Ohms, charges are in the units of Coulombs, and capacitances are measured in Coulombs per Volt.

The following sections deal with uncertainty quantification and verification of the Inverse FORM approach.

\section{Uncertainty Quantification in RUL}

In this example, the process noise of the state space model is assumed to be small as noted by Daigle et al. [17], and therefore not included in the uncertainty quantification. The two types of uncertainty considered are:

1. Loading Uncertainty: In this example, a constant amplitude loading is considered for the purpose of illustration. The amplitude is considered to be normally distributed $(N(1.375,1 / 6))$, and this distribution is truncated at a specified lower bound (0.75) and an upper bound (2.00).

2. State Uncertainty: Typically, the state estimation, which is an inverse problem, is addressed using a filtering technique which can continuously estimate the uncertainty in the state when measurements are continuously available as a function of time. In this paper, the state estimation is not explicitly carried out. The state values are assumed to be available, and the uncertainty in the states are predetermined based on the authors' past experiences with the use of filtering techniques for the above described problem. There are two state variables (charge in the battery and charge associated with the capacitance) in this example and at any time instant, they are assumed to be normally distributed with a specified mean; for example, the mean of the initial states are set as $\left[4.14 \times 10^{4}, 0\right]$. For the purpose of illustration, three different values of $\mathrm{CoV}$ (Coefficient of variation, defined as the ratio 


\begin{tabular}{c|ccc}
\hline & CoV=1\% & CoV=2\% & CoV=3\% \\
\hline \multirow{2}{*}{$\mathbf{T = 0}$} & $r_{1}=4065$ & $r_{1}=3781$ & $r_{1}=3401$ \\
& $r_{2}=5045$ & $r_{2}=5045$ & $r_{2}=5045$ \\
& $r_{3}=6459$ & $r_{3}=6742$ & $r_{3}=7120$ \\
\hline \multirow{2}{*}{$\mathbf{T = 1 0 0 0}$} & $r_{1}=3216$ & $r_{1}=2908$ & $r_{1}=2515$ \\
& $r_{2}=4045$ & $r_{2}=4045$ & $r_{2}=4045$ \\
& $r_{3}=5226$ & $r_{3}=5532$ & $r_{3}=5924$ \\
\hline \multirow{2}{*}{$\mathbf{T = 2 0 0 0}$} & $r_{1}=2359$ & $r_{1}=2023$ & $r_{1}=1616$ \\
& $r_{2}=3045$ & $r_{2}=3045$ & $r_{2}=3045$ \\
& $r_{3}=4001$ & $r_{3}=4335$ & $r_{3}=4741$ \\
\hline \multirow{2}{*}{$\mathbf{T = 3 0 0 0}$} & $r_{1}=1488$ & $r_{1}=1118$ & $r_{1}=699$ \\
& $r_{2}=2045$ & $r_{2}=2045$ & $r_{2}=2045$ \\
& $r_{3}=2789$ & $r_{3}=3157$ & $r_{3}=3575$ \\
\hline \multirow{2}{*}{$\mathbf{T = 4 0 0 0}$} & $r_{1}=589$ & $r_{1}=186$ & $r_{1}=43$ \\
& $r_{2}=1045$ & $r_{2}=1045$ & $r_{2}=1045$ \\
& $r_{3}=1605$ & $r_{3}=2008$ & $r_{3}=2432$ \\
\hline \multirow{2}{*}{$\mathbf{T = 5 0 0 0}$} & $r_{1}=6$ & $r_{1}=1$ & $r_{1}=0$ \\
& $r_{2}=45$ & $r_{2}=45$ & $r_{2}=45$ \\
& $r_{3}=478$ & $r_{3}=897$ & $r_{3}=1316$ \\
\hline & \multicolumn{3}{c}{}
\end{tabular}

Table 2: Results of Uncertainty Quantification in RUL

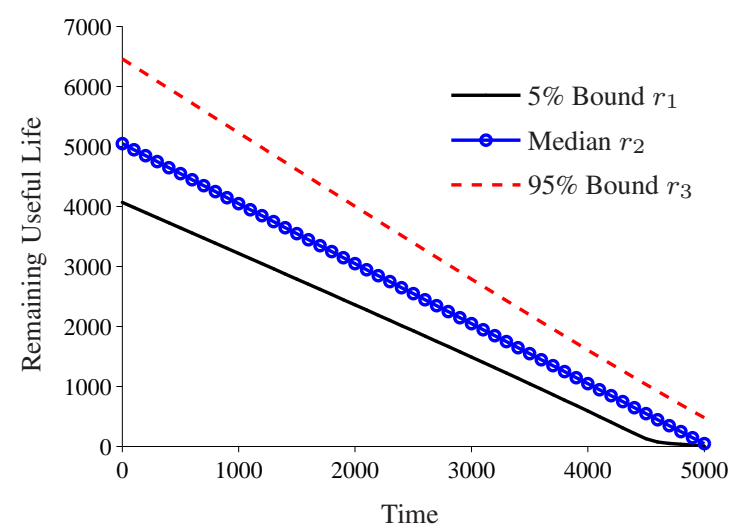

Figure 3: $90 \%$ Bounds and Median of RUL $(\mathrm{CoV}=1 \%)$

between standard deviation and mean) are considered $0.01,0.02,0.03$ - and the analysis is repeated for each $\mathrm{CoV}$ value.

In order to apply the proposed algorithm to this battery example, the first step is to construct the " $G$ " model as seen earlier in Eq. 6. One realization of each source of uncertainty (two variables corresponding to the two states in this example, and one variable corresponding to the loading) becomes input to this model, and the state space equations above are used to calculate the remaining useful life prediction (right hand side of Eq. 6). Then, the Inverse FORM method can be directly used to compute the RUL value corresponding to multiple CDF values; $F_{R}\left(r_{1}\right)=0.05, F_{R}\left(r_{2}\right)=0.5$, and $F_{R}\left(r_{3}\right)=0.95$ are used in this paper, and the calculations are repeated for three considered $\mathrm{CoV}$ values for the uncertainty in the state variables. While $r_{1}$ and $r_{3}$ correspond to the $90 \%$ probability bounds of RUL, $r_{2}$ corresponds the median of RUL. The bounds and the mean are continuously calculated until $T=5000$ (in seconds) when failure seems to be imminent, and the Inverse FORM calculation is performed every $100 \mathrm{~s}$. The results are tabulated in Table 2 and graphically shown in Fig. 3-5.

It is seen that from the results that the uncertainty in the RUL is high initially, and then gradually decreases until failure is imminent. Initially, the uncertainty in RUL is high because it is necessary to predict at a further time instant, in comparison with making prognostic predictions at a later stage. In fact, any good prognostic algorithm should depict this behavior, i.e., the prediction of RUL at a later time instant has lower

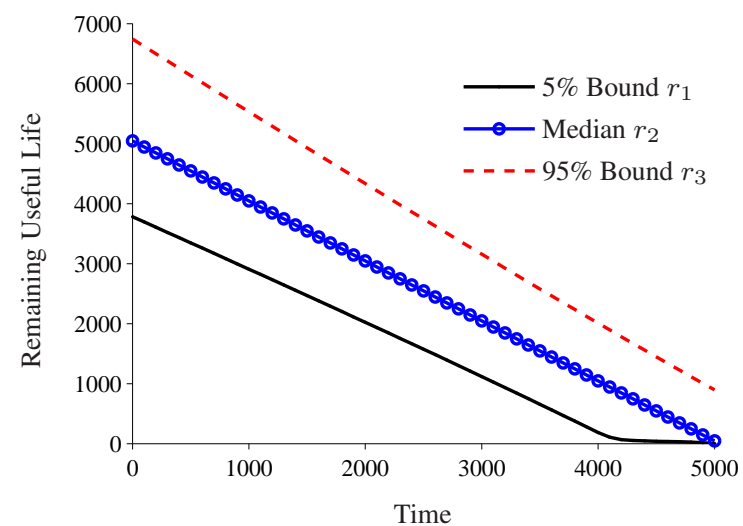

Figure 4: $90 \%$ Bounds and Median of RUL $(\mathrm{CoV}=2 \%)$

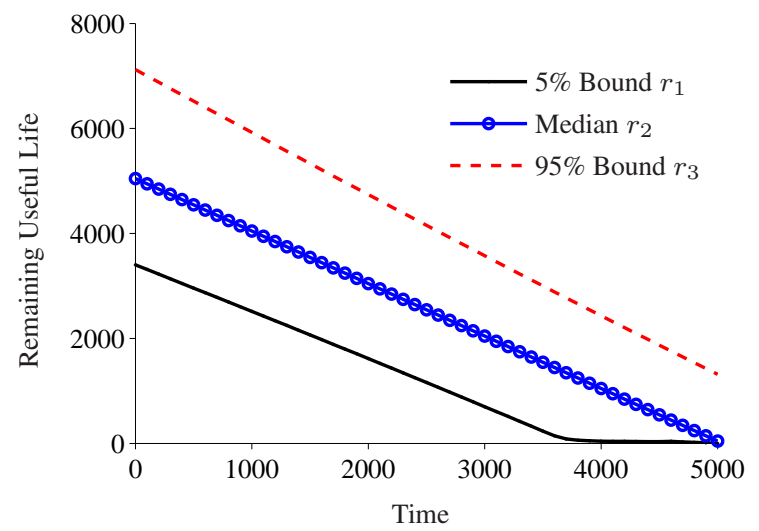

Figure 5: 90\% Bounds and Median of RUL: $(\mathrm{CoV}=3 \%)$

uncertainty than the prediction at an earlier time instant. Further, the larger the coefficient of variation, the larger the uncertainty in RUL; this behavior is observed at every time instant and is consistent with intuition because a larger uncertainty in the state estimate implies that the corresponding RUL prediction uncertainty will also be high. When failure is imminent, the probability distribution of RUL is highly positively skewed; in other words, the probability density function has a very high likelihood for almost immediate failure, but has a longer right-tail. The maximum likelihood point corresponds to the time of imminent failure, while the variance is reflective of the various sources of uncertainty.

\section{Verification using Monte Carlo Sampling}

In order to verify the above performed uncertainty quantification, Monte Carlo sampling (MCS) is performed using 1000 samples. It is computationally infeasible to perform MCS at every time instant considered. Therefore, MCS is performed for six time instants, starting from $T=0$ until $T=5000$ in steps of 1000 seconds. At each time instant, the CDF of RUL was computed by repeating Inverse FORM for 13 different $\lambda$ values $(\lambda=0.01,0.05,0.1,0.2, \ldots 0.8,0.9,0.95,0.99)$. The comparison of Inverse FORM and MCS at $T=0$ is shown in Fig. 6; in this illustration, $\mathrm{CoV}$ of state estimates is chosen to be $3 \%$. Note that the uncertainty bounds due to the use of limited number of samples for MCS are also shown.

It is seen that the probability distribution resulting from Inverse FORM lies within the Monte Carlo bounds, as seen in Fig. 6, thus verifying the uncertainty quantification procedure. In fact, the maximum difference between the Inverse 


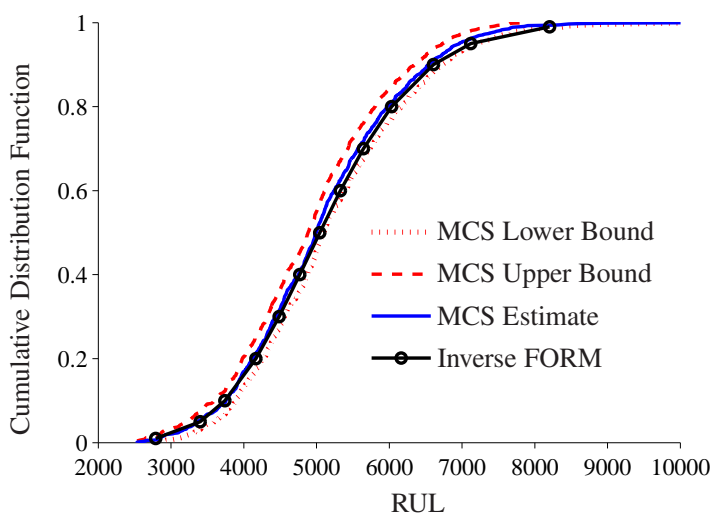

Figure 6: Inverse FORM vs. MCS ( $T=0, C o V=3 \%)$

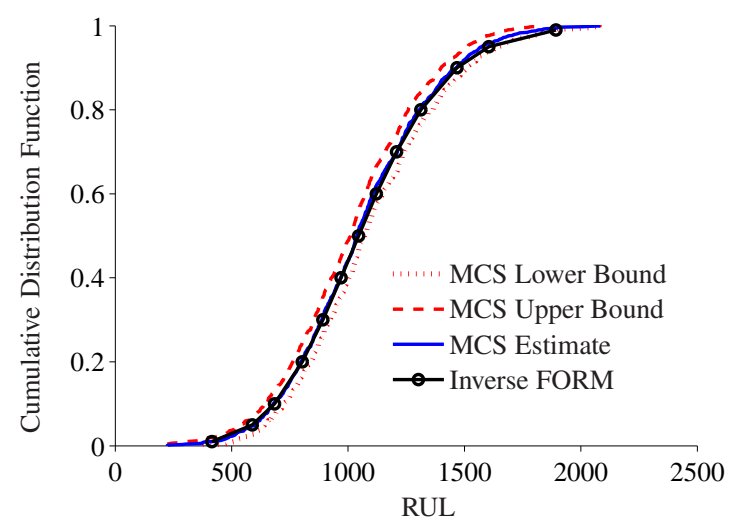

Figure 7: Inverse FORM vs. MCS ( $T=4000, C o V=1 \%)$

FORM solution and the Monte carlo solution was found to be less than $0.5 \%$ (it must be noted that the Monte carlo solution is not exact due to the use of limited samples and hence, the $90 \%$ Monte Carlo bounds have also been provided in Fig. 6). Further, the Inverse FORM procedure needed only $13 \times 4 \times 3=156$ prognostic evaluations, since $13 \lambda$ values and 3 variables (to represent uncertain quantities) were used, and 4 iterations were needed for convergence whenever the iterative Inverse FORM algorithm was used. In contrast, MCS required 1000 prognostic evaluations. Further, a similar comparison was performed at different time steps, and by considering other $\mathrm{CoV}$ values. For instance, the comparison of the results from MCS and Inverse FORM at $T=4000 \mathrm{~s}$ for $\mathrm{CoV}=1 \%$ is shown in Fig. 7 .

The agreement of Inverse FORM with MCS is evident in Fig. 7. In fact, it was observed that the Inverse FORM method performs well in comparison with Monte Carlo sampling, both in terms of accuracy (the maximum difference was found to be less than 1\%) and computational cost (approximately $10 \%$ of the cost of MCS).

\section{Conclusion}

Conventionally, sampling-based algorithms have been used for quantifying the uncertainty in prognostic calculations, and may require several thousands of samples in order to quantify the entire probability distribution of remaining useful life (RUL) with reasonable accuracy. Further, if such an algorithm were to be repeated, a probability distribution different (albeit only slightly) from the original distribution may be obtained, due to the use of limited samples. These two reasons hinder the use of such algorithms in online prognostics and decision-making. Therefore, this paper investigated the use of analytical algorithms in order to quantify the uncertainty in the RUL prediction.

The First-Order Second Moment method (FOSM), the FirstOrder Reliability Method (FORM), and Inverse First-Order Reliability Method (Inverse FORM) are optimization-based analytical approaches, which were originally developed by structural engineers in order to quantify the reliability of structural systems. This paper extended these methods to quantify the entire probability distribution of RUL using state-space models that capture the evolution of the system continuously as a function of time. While the FOSM method can be used to obtain the first two moments of the RUL prediction, the FORM and Inverse FORM approaches can be used to calculate the entire CDF of the RUL. These approaches are not only computationally cheaper, but can produce repeatable results, and therefore, are suitable for online prognosis and decision-making.

Future work needs to address several issues. First, the process noise of the state space models need to be rigorously accounted for in the uncertainty quantification procedure. This is particularly important when the contribution of process noise uncertainty to the overall uncertainty in RUL is significantly large. Second, practical systems are commonly subjected to different types of variable amplitude loading profiles such as block loading, Markov processes, general random processes, etc., and therefore, the proposed methods for uncertainty quantification need to be extended to consider variable amplitude loading. The assumption of constant amplitude loading implies that loading uncertainty is described using a single random variable, whereas variable amplitude loading profiles need to be described using multiple random variables, which not only increases the dimensionality of the problem, but also affects the uncertainty in the RUL prediction. Future work must investigate the impact of including variable amplitude loading on the uncertainty bounds of the RUL prediction. Third, sensitivity analysis needs to be performed so that the contributions of the different sources of uncertainty to the overall uncertainty in RUL can be quantified. Fourth, this paper did not consider the effect of model form uncertainty on prognosis; future research needs to quantify model form uncertainty and develop a method to rigorously account for model uncertainty in prognosis and RUL calculations. Finally, it is also necessary to quantify the robustness of the proposed approach, by estimating the sensitivity of the RUL bounds to relaxing the various assumptions in the present paper, and thereby investigate the applicability of the methodology to practical engineering systems.

\section{ACKNOWLEDGMENTS}

The work reported herein was in part funded by NASA ARMD/AvSafe project SSAT and by NASA/OCT/AES project ACLO. The support is gratefully acknowledged.

\section{REFERENCES}

[1] J. Luo, K. Pattipati, L. Qiao, and S. Chigusa, "Modelbased prognostic techniques applied to a suspension system," Systems, Man and Cybernetics, Part A: Systems and Humans, IEEE Transactions on, vol. 38, no. 5, 
pp. 1156-1168, 2008.

[2] K. Goebel, B. Saha, and A. Saxena, "A comparison of three data-driven techniques for prognostics," in 62nd Meeting of the Society For Machinery Failure Prevention Technology (MFPT), 2008, pp. 119-131.

[3] M. Daigle, M. Foygel, and V. Smelyanskiy, "Modelbased diagnostics for propellant loading systems," in Aerospace Conference, 2011 IEEE. IEEE, 2011, pp. $1-11$.

[4] M. Daigle and K. Goebel, "A model-based prognostics approach applied to pneumatic valves," International Journal of Prognostics and Health Management, vol. 2, no. 2, 2011.

[5] S. Sankararaman and S. Mahadevan, "Uncertainty quantification in structural damage diagnosis," Structural Control and Health Monitoring, vol. 18, no. 8, pp. 807-824, 2011.

[6] _ - "Bayesian methodology for diagnosis uncertainty quantification and health monitoring," Structural Control and Health Monitoring, vol. In Press, 2011.

[7] S. Sankararaman, Y. Ling, C. Shantz, and S. Mahadevan, "Uncertainty quantification in fatigue damage prognosis," in Annual Conference of the Prognostics and Health Management Society, 2009.

[8] —, "Uncertainty quantification in fatigue crack growth prognosis," International Journal of Prognostics and Health Management, vol. 2, no. 1, 2011.

[9] C. Farrar and N. Lieven, "Damage prognosis: the future of structural health monitoring," Philosophical Transactions of the Royal Society A: Mathematical, Physical and Engineering Sciences, vol. 365, no. 1851, pp. 623632, 2007.

[10] B. Saha and K. Goebel, "Uncertainty management for diagnostics and prognostics of batteries using bayesian techniques," in Aerospace Conference, 2008 IEEE. IEEE, 2008, pp. 1-8.

[11] X. Guan, Y. Liu, R. Jha, A. Saxena, J. Celaya, and K. Geobel, "Comparison of two probabilistic fatigue damage assessment approaches using prognostic performance metrics," International Journal of the PHM Society, vol. 1, p. 005, 2011.

[12] P. Djuric, J. Kotecha, J. Zhang, Y. Huang, T. Ghirmai, M. Bugallo, and J. Miguez, "Particle filtering," Signal Processing Magazine, IEEE, vol. 20, no. 5, pp. 19-38, 2003.

[13] D. Calvetti and E. Somersalo, Introduction to Bayesian scientific computing: Ten lectures on subjective computing. Springer New York, 2007, vol. 2.

[14] B. Saha, C. C. Quach, and K. Goebel, "Optimizing battery life for electric UAVs using a Bayesian framework," in Proceedings of the 2012 IEEE Aerospace Conference, March 2012.

[15] Y. Ling, C. Shantz, S. Mahadevan, and S. Sankararaman, "Stochastic prediction of fatigue loading using real-time monitoring data," International Journal of Fatigue, vol. 33, no. 7, pp. 868 - 879, 2011.

[16] J. Celaya, A. Saxena, and K. Goebel, "Uncertainty representation and interpretation in model-based prognostics algorithms based on kalman filter estimation," in Annual Conference of the Prognostics and Health Management Society, 2012.

[17] M. Daigle, A. Saxena, and K. Goebel, “An efficient deterministic approach to model-based prediction uncertainty estimation," in Annual Conference of the Prognostics and Health Management Society, 2012.

[18] A. Haldar and S. Mahadevan, Probability, reliability, and statistical methods in engineering design. John Wiley \& Sons, Inc., 2000.

[19] B. Fiessler, R. Rackwitz, and H. Neumann, "Quadratic limit states in structural reliability," Journal of the Engineering Mechanics Division, vol. 105, no. 4, pp. 661676, 1979.

[20] X. Chen and N. Lind, "Fast probability integration by three-parameter normal tail approximation," Structural Safety, vol. 1, no. 4, pp. 269-276, 1983.

[21] E. Balaban, S. Narasimhan, M. Daigle, J. Celaya, I. Roychoudhury, B. Saha, S., and K. Goebel, "A mobile robot testbed for prognostics-enabled autonomous decision making," in Annual Conference of the Prognostics and Health Management Society, 2011.

[22] M. Chen and G. A. Rincon-Mora, "Accurate electrical battery model capable of predicting runtime and I-V performance," IEEE Transactions on Energy Conversion, vol. 21, no. 2, pp. 504 - 511, jun 2006.

\section{BIOGRAPHY}

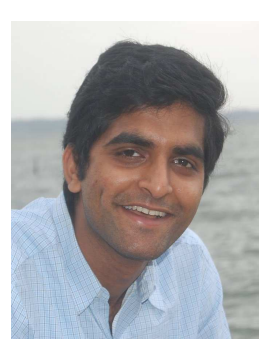

Shankar Sankararaman received his B.S. degree in Civil Engineering From Indian Institute of Technology, Madras in India in 2007 and recently obtained his Ph.D. in Civil Engineering at Vanderbilt University, Nashville, Tennessee, U.S.A in 2012. His dissertation titled "Uncertainty Quantification and Integration in Engineering Systems" focuses on the various aspects of uncertainty quantification in civil, mechanical, and aerospace systems. His research interests include probabilistic methods, risk and reliability analysis, Bayesian networks, structural health monitoring, diagnosis and prognosis, decision-making under uncertainty, treatment of epistemic uncertainty, and multidisciplinary analysis. He is a member of Probabilistic Methods Technical Committee (PMC) at ASCE and a member of Non-Deterministic Approaches (NDA) technical committee at AIAA. Currently, Shankar is a post-doctoral research scholar with Stinger and Ghaffarian Technologies at NASA Ames Research Center, Moffett field, CA, where he develops algorithms for uncertainty assessment and management in prognostics and decision-making.

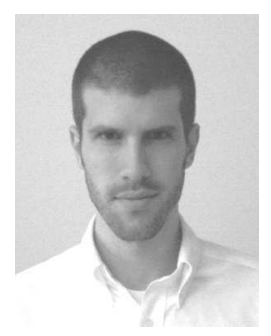

Matthew Daigle received the B.S. degree in Computer Science and Computer and Systems Engineering from Rensselaer Polytechnic Institute, Troy, NY, in 2004, and the M.S. and Ph.D. degrees in Computer Science from Vanderbilt University, Nashville, TN, in 2006 and 2008, respectively. From September 2004 to May 2008, he was a Graduate Research Assistant with the Institute for Software Integrated Systems and Department of Electrical Engineering and Computer Science, Vanderbilt University, Nashville, TN. During the summers of 2006 and 2007, he was an intern with Mission Critical Technologies, Inc., at NASA Ames Research Center. From June 2008 to December 2011, 
he was an Associate Scientist with the University of California, Santa Cruz, at NASA Ames Research Center. Since January 2012, he has been with NASA Ames Research Center as a Research Computer Scientist. His current research interests include physics-based modeling, model-based diagnosis and prognosis, simulation, and hybrid systems.

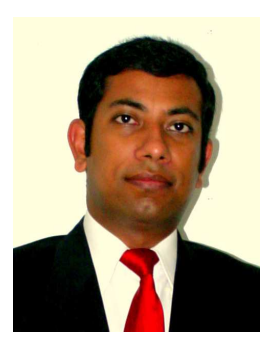

Abhinav Saxena is a Research Scientist at the Prognostics Center of Excellence, NASA Ames Research Center, Moffett Field CA. His research focus lies in developing and evaluating prognostic algorithms for engineering systems including electromechanical actuators, electronic semiconductor devices, and power storage devices. These algorithms apply various classification, regression and state estimation techniques for predicting remaining useful life of systems and their components. He is also working on developing systems engineering process and methods for verification and validation for health management systems. He has published over fifty research papers on these topics. $\mathrm{He}$ is currently the chief editor of the International Journal of Prognostics and Health Management (IJPHM). He has a $\mathrm{PhD}$ in Electrical and Computer Engineering from Georgia Institute of Technology, Atlanta. He earned his B.Tech in 2001 from Indian Institute of Technology (IIT) Delhi, and Masters Degree in 2003 from Georgia Tech. Abhinav has been a GM manufacturing scholar and is also a member of IEEE, AAAI and ASME.

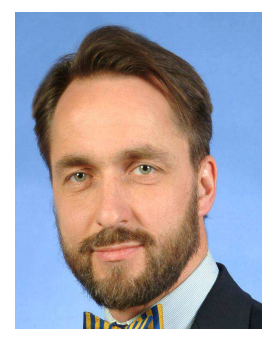

Kai Goebel is the Deputy Area Lead for Discovery and Systems Health at NASA Ames where he also directs the Prognostics Center of Excellence. After receiving the $\mathrm{Ph}$.D. from the University of California at Berkeley in 1996, Dr. Goebel worked at General Electrics Corporate Research Center in Niskayuna, NY from 1997 to 2006 as a senior research scientist before joining NASA.

He has carried out applied research in the areas of artificial intelligence, soft computing, and information fusion and his interest lies in advancing these techniques for real time monitoring, diagnostics, and prognostics. He holds fifteen patents and he has published more than 200 papers in the area of systems health management. 